\title{
Concept innovant de l'Interface à Collision et Réaction (CRI) pour la gestion des interférences polyatomiques en ICP-MS. Application au dosage multi élémentaire dans les matrices biologiques
}

\section{Innovative concept of the Collison Reaction Interface (CRI) for polyatomic Interferences management by ICP-MS. Application to multi-elemental analysis in biological matrices}

Yolande ABDELNOUR

VARIAN S.A. 7, avenue des Tropiques, Z.A. de Courtabœuf 2 - B.P. 12, 91941 Les ULIS Cedex, France.

(Reçu le 23 février 2007 ; accepté après modifications le 27 avril 2007)

\section{RÉSUMÉ}

L'analyse d'éléments à l'état de traces dans les fluides biologiques par ICP-MS s'est largement développée au cours des dernières années. Cette technique offre plusieurs avantages par rapport à l'absorption atomique utilisée traditionnellement, notamment la possibilité d'analyse multi élémentaire, la préparation simplifiée des échantillons et l'amélioration des limites de détection. Cependant, les performances d'un analyseur de masse quadripolaire peuvent être compromises par la présence d'interférences spectroscopiques qui empêchent le dosage de certains éléments "difficiles » comme le chrome, l'arsenic, le sélénium... à faibles teneurs. Le présent article décrit le concept innovant de gestion des interférences à travers l'Interface à Réaction et à Collision CRI* (brevet Varian) intégré au modèle 820-MS CRI-ICP-MS VARIAN ainsi que son utilisation dans

\section{SUMMARY}

Inductively Coupled Plasma Mass Spectrometry (ICP-MS) has been extensively used over the past few years for the determination of trace and ultra-trace elements in biological samples. This technique offers multi-elemental capabilities, ease of use, simplified sample preparation and improved detection limits over traditional atomic spectroscopy techniques. However, the performances of traditional quadrupole mass analyzers can be compromised by the presence of several spectral interferences which deteriorates the detection capability for some critical elements like chromium, arsenic, selenium. This paper describes a novel approach for interference removal via the use of patented collision reaction interface (CRI) integrated in the Varian 820-MS CRI-ICP-MS and applying it to the simultaneous 
l'analyse simultanée d'éléments traces et majeurs dans les matrices biologiques comme le sang et l'urine.

\section{MOTS-CLÉS}

Interface, collision, réaction, spectrométrie de masse, plasma inductif, interférences, matrices biologiques.

\section{Introduction}

L'analyse élémentaire dans les fluides biologiques par spectrométrie de masse quadripolaire couplée à une torche à plasma inductif (ICP-MS) s'est largement développée au cours des dernières années $(1,2)$. Cette technique offre en effet plusieurs avantages par rapport aux techniques d'absorption atomique, flamme et four utilisées traditionnellement : la possibilité d'effectuer des dosages multi-élémentaires, l'obtention de faibles limites de détection et une préparation simple de l'échantillon, limitée dans la plupart des cas à une dilution avant l'analyse... Cependant, les performances de l'ICP-MS quadripôlaire peuvent être compromises par la présence d'interférences. Celles-ci peuvent être non spectroscopiques (viscosité de l'échantillon), chimiques (interaction entre les éléments de la matrice) et spectroscopiques $(3,4)$ (recouvrements d'ions isobariques et/ou polyatomiques). $\mathrm{La}$ formation d'interférences polyatomiques peut provenir des ions du plasma, du solvant ou de la matrice de l'échantillon. Les tableaux $1 \mathrm{a}$ et $1 \mathrm{~b}$ regroupent quelques interférences potentielles provenant du plasma (tableau 1a) et rencontrées dans les milieux biologiques tels que sang et urine (tableau 1b).

Tableau Ia : Interférences liées au plasma.

\begin{tabular}{|l|c|c|}
\hline \multicolumn{1}{|c|}{ Interférent } & $\mathbf{M} / \mathbf{Z}$ & Ion analyte \\
\hline${ }^{16} \mathrm{O}_{2}^{+}$ & 32 & $\mathrm{~S}^{+}$ \\
\hline${ }^{16,18} \mathrm{O}_{2}^{+}$ & 34 & $\mathrm{~S}^{+}$ \\
\hline${ }^{40} \mathrm{Ar}^{+}$ & 40 & $\mathrm{Ca}^{+}$ \\
\hline${ }^{40} \mathrm{ArH}^{+}$ & 41 & $\mathrm{~K}^{+}$ \\
\hline${ }^{40} \mathrm{ArH}_{2}^{+}$ & 42 & $\mathrm{Ca}^{+}$ \\
\hline${ }^{12} \mathrm{C}^{16} \mathrm{O}_{2}^{+}$ & 44 & $\mathrm{Ca}^{+}$ \\
\hline${ }^{40} \mathrm{Ar}^{12} \mathrm{C}^{+}$ & 52 & $\mathrm{Cr}^{+}$ \\
\hline${ }^{40} \mathrm{Ar}^{14} \mathrm{~N}^{+}$ & 54 & $\mathrm{Fe}^{+}, \mathrm{Cr}^{+}$ \\
\hline${ }^{40} \mathrm{Ar}^{16} \mathrm{O}^{+}$ & 56 & $\mathrm{Fe}^{+}$ \\
\hline${ }^{40} \mathrm{Ar}^{16} \mathrm{OH}^{+}$ & 57 & $\mathrm{Fe}^{+}$ \\
\hline${ }^{40} \mathrm{Ar}^{18} \mathrm{O}^{+}$ & 58 & $\mathrm{Ni}^{+}, \mathrm{Fe}^{+}$ \\
\hline${ }^{40} \mathrm{Ar}^{12} \mathrm{C}^{16} \mathrm{O}^{+}$ & 68 & $\mathrm{Zn}^{+}$ \\
\hline${ }^{36,40} \mathrm{Ar}_{2}^{+}$ & 76 & $\mathrm{Se}^{+}$ \\
\hline${ }^{38,40} \mathrm{Ar}_{2}^{+}$ & 78 & $\mathrm{Se}^{+}$ \\
\hline${ }^{40,40} \mathrm{Ar}_{2}^{+}$ & 80 & $\mathrm{Se}^{+}$ \\
\hline
\end{tabular}

analysis of trace and major elements in biological matrices such as whole blood and urine.

\section{KEY-WORDS}

Interface, collision, reaction, mass spectrometry, inductively coupled plasma, interferences, biological matrices.

Tableau Ib : Interférences liées aux matrices biologiques.

\begin{tabular}{|c|c|c|c|c|}
\hline Éléments & Hydrures & Oxydes & Argide & Doublement \\
\hline${ }^{12} \mathrm{C}^{+}$ & & & ${ }^{52} \mathrm{Cr}^{+}$ & \\
\hline${ }^{23} \mathrm{Na}^{+}$ & ${ }^{24} \mathrm{Mg}^{+}$ & ${ }^{39} \mathrm{~K}^{+}$ & ${ }^{63} \mathrm{Cu}^{+}$ & ${ }^{12} \mathrm{C}^{+}$ \\
\hline${ }^{24} \mathrm{Mg}^{+}$ & ${ }^{25} \mathrm{Mg}^{+}$ & ${ }^{40} \mathrm{Ca}^{+, 40} \mathrm{~K}^{+}$ & ${ }^{64} \mathrm{Ni}^{+}$ & ${ }^{12} \mathrm{C}^{+}$ \\
\hline${ }^{25} \mathrm{Mg}^{+}$ & ${ }^{26} \mathrm{Mg}^{+}$ & ${ }^{41} \mathrm{~K}^{+}$ & ${ }^{65} \mathrm{Cu}^{+}$ & ${ }^{12} \mathrm{C}^{+, 13} \mathrm{C}^{+}$ \\
\hline${ }^{26} \mathrm{Mg}^{+}$ & ${ }^{27} \mathrm{Al}^{+}$ & ${ }^{42} \mathrm{Ca}^{+}$ & ${ }^{66} \mathrm{Zn}^{+}$ & ${ }^{13} \mathrm{C}^{+}$ \\
\hline${ }^{39} \mathrm{~K}^{+}$ & ${ }^{40} \mathrm{Ca}^{+}$ & ${ }^{55} \mathrm{Mn}^{+}$ & ${ }^{79} \mathrm{Se}^{+}$ & \\
\hline${ }^{35} \mathrm{Cl}^{+}$ & & ${ }^{51} \mathrm{~V}^{+}$ & ${ }^{75} \mathrm{As}^{+}$ & \\
\hline${ }^{40} \mathrm{~K}^{+}$ & ${ }^{41} \mathrm{~K}^{+}$ & ${ }^{56} \mathrm{Fe}^{+}$ & ${ }^{80} \mathrm{Se}^{+}$ & \\
\hline${ }^{41} \mathrm{~K}^{+}$ & ${ }^{42} \mathrm{Ca}^{+}$ & ${ }^{57} \mathrm{Fe}^{+}$ & & \\
\hline${ }^{40} \mathrm{Ca}^{+}$ & ${ }^{41} \mathrm{~K}^{+}$ & ${ }^{56} \mathrm{Fe}^{+}$ & ${ }^{80} \mathrm{Se}^{+}$ & \\
\hline${ }^{42} \mathrm{Ca}^{+}$ & ${ }^{43} \mathrm{Ca}^{+}$ & ${ }^{58} \mathrm{Fe}^{+}$ & ${ }^{82} \mathrm{Se}^{+}$ & \\
\hline${ }^{43} \mathrm{Ca}^{+}$ & ${ }^{44} \mathrm{Ca}^{+}$ & ${ }^{59} \mathrm{Co}^{+}$ & & \\
\hline${ }^{44} \mathrm{Ca}^{+}$ & ${ }^{45} \mathrm{Sc}^{+}$ & ${ }^{60} \mathrm{Ni}^{+}$ & & \\
\hline${ }^{54} \mathrm{Fe}^{+}$ & ${ }^{55} \mathrm{Mn}^{+}$ & ${ }^{70} \mathrm{Zn}^{+}$ & & ${ }^{27} \mathrm{Al}^{+}$ \\
\hline $\begin{array}{l}{ }^{56} \mathrm{Fe}^{+} \\
\end{array}$ & ${ }^{57} \mathrm{Fe}^{+}$ & & & \\
\hline${ }^{57} \mathrm{Fe}^{+}$ & ${ }^{58} \mathrm{Fe}^{+}$ & & & \\
\hline${ }^{58} \mathrm{Fe}^{+}$ & ${ }^{59} \mathrm{Co}^{+}$ & & & \\
\hline
\end{tabular}

\section{Matériel et Méthodes}

Plusieurs techniques conventionnelles ont été développées pour la gestion des interférences polyatomiques en ICP-MS, telles que : utilisation d'équations de correction mathématiques, élimination de la matrice, technique de plasma froid ou utilisation de spectromètres haute résolution (5). Le développement au cours des dix dernières années des systèmes de collisions et de réactions pour la gestion des interférences a permis d'élargir les capacités de l'ICP-MS. Ces systèmes sont basés sur l'ajout de gaz (inertes ou réactifs : $\mathrm{He}, \mathrm{CH}_{4}$, $\left.\mathrm{NH}_{3}, \mathrm{H}_{2} \ldots\right)$ dans un multipôle placé entre l'optique ionique et l'entrée de l'analyseur de masse. L'ajout du gaz permet, par des mécanismes de collisions et/ou de réactions de réduire l'interférence spectroscopique.

L'interface à collision et à réaction CRI* (Brevet VARIAN) est un concept unique au 820-MS permettant la gestion simple et efficace des interférences polyatomiques. L'injection de gaz tels que $\mathrm{H}_{2}$ et/ou He s'effectue dans le plasma lors de son passage à travers les orifices des cônes (figure I). La température et la densité du plasma au niveau des cônes favorisent les réactions de transfert de charge, les interactions électrons - ions et ions - molécules afin d'éliminer de façon instantanée les interférents polyatomiques. Les lentilles d'extraction placées à l'arrière du cône écréteur permettent également la discrimination en énergie des 


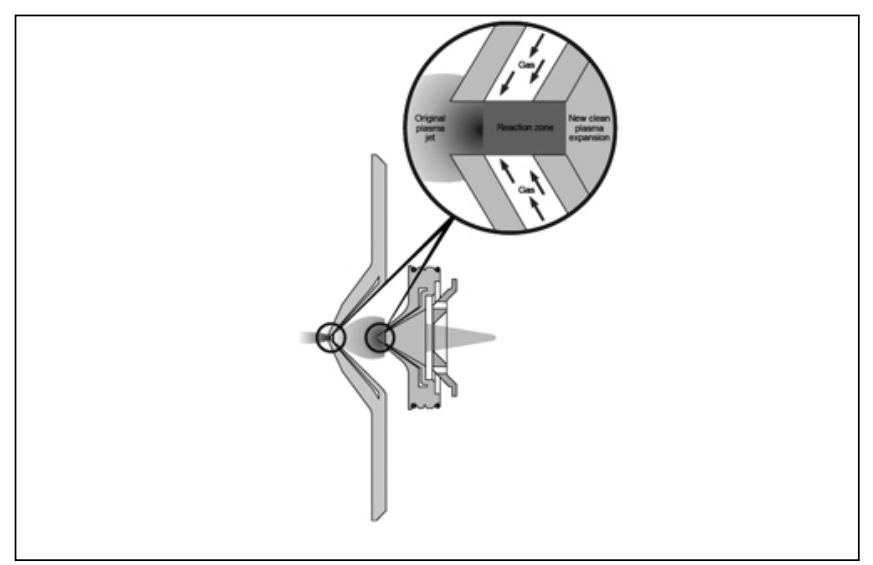

Figure 1 : Concept CRI pour la gestion des interférences polyatomiques.

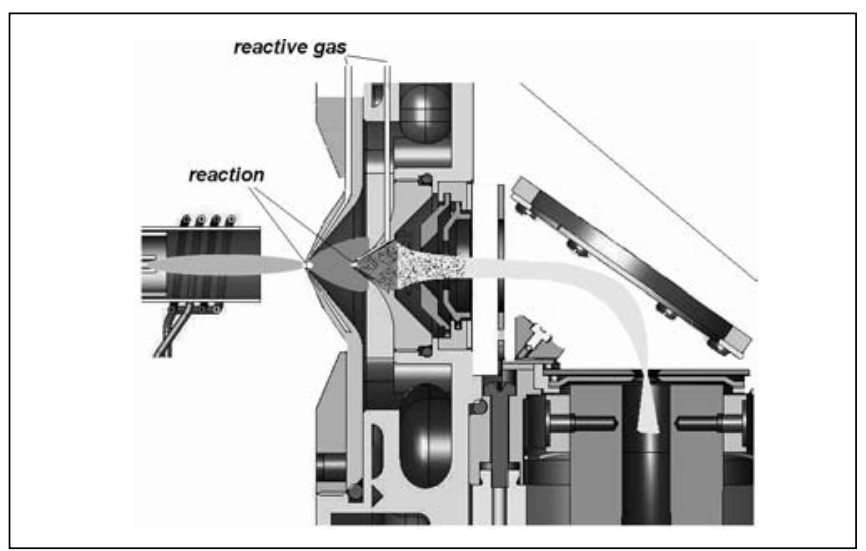

Figure 2 : Schéma d'ensemble du 820-MS.

ions pour une gestion simple et efficace de l'ensemble des espèces interférentes. L'efficacité de pompage à l'interface permet d'assurer un passage rapide entre les modes standard (sans gaz) et CRI (avec $\mathrm{H}_{2}$ et/ou $\mathrm{He}$ ) pour une productivité maximale et une analyse complète de l'ensemble des éléments.

Le nouveau concept de l'optique ionique à $90^{\circ}$ (Brevet Varian $^{7}$ ) présent dans la gamme ICP-MS VARIAN 810MS et 820-MS (figure II) permet d'obtenir une sensibilité supérieure au Giga Hertz (1000 Million cps/ppm) tout en maintenant des taux d'oxydes $(\mathrm{CeO} / \mathrm{Ce}<1 \%)$ et de doublement chargés $\left(\mathrm{Ce}^{++} / \mathrm{Ce}^{+}<2 \%\right)$ faibles.

La haute sensibilité permet non seulement d'obtenir des limites de détection très basses mais également d'avoir une flexibilité quant au choix de l'isotope, du mode de fonctionnement selon le type d'échantillon ou l'application en cours.

Le détecteur est de type multiplicateur d'électrons à dynodes discrètes DDEM (Discrete Dynode Electron Multiplier). Il assure une gamme linéaire dynamique de plus de 9 ordres de grandeurs et ce en mode comptage de coups uniquement. Ceci permet de s'affranchir des problèmes d'étalonnages croisés observés avec les détecteurs conventionnels lors du passage en mode analogique pour les éléments à fortes teneurs.

L'ICP-MS 820-MS de VARIAN est utilisé pour les analyses de sang total et d'urine. Le système d'introduction est un nébuliseur MicroMist $0,4 \mathrm{ml} / \mathrm{min}$ (Glass Expansion USA), et la chambre de nébulisation de type Scott double passe refroidie par effet Peltier à $3^{\circ} \mathrm{C}$. Tous les paramètres instrumentaux sont pilotés par ordinateur.

Le logiciel ICP-MS Expert permet d'effectuer des optimisations automatiques des paramètres du plasma, de l'optique ionique ainsi que des débits de gaz CRI pour l'obtention des meilleures performances (Meilleure sensibilité, taux d'oxydes et de doublement chargés les plus faibles).

L'analyse de l'ensemble des éléments traces et majeurs a été effectuée avec des conditions opératoires identiques pour l'ensemble des isotopes, en mode CRI avec le gaz $\mathrm{H}_{2}$ introduit au niveau du cône écréteur à un débit de $0,1 \mathrm{~L} / \mathrm{min}$. L'utilisation du gaz $\mathrm{H}_{2}$ permet de gérer les interférences polyatomiques sur des éléments difficiles tels que As, $\mathrm{Se}, \mathrm{V}$ et $\mathrm{Cr}$ sans compromettre les performances pour les éléments non interférés. L'utilisation de conditions opératoires identiques pour tous les éléments présente l'avantage de réduire le temps d'analyse global et, par conséquent, le volume d'échantillon consommé.

\section{Réactifs}

Les échantillons de Seronorm sang total WB, urine 02525 sont fournis par la société SERO AS (Norvège). L'échantillon urine 2670a est fourni par le «National Institute of Standard \& Technology (NIST). Le TritonX100 est fourni par BDH Chemicals). L'ammonium EDTA (ethylene diamine tetracetic acid, sel diammonium) de pureté $97 \%$ est fourni par la société Aldrich. La solution mère multi-élémentaire S28 à $100 \mu \mathrm{g} \mathrm{mL}-1$ est fournie par la société Carlo Erba.

\section{Préparation des solutions}

\section{Échantillon :}

Les échantillons lyophilisés sont reconstitués comme recommandé par le fournisseur.

Sang total : Les échantillons de sang total sont dilués par un facteur 1:10 avant analyse. La solution de diluant est constituée de : 0,1\% Triton-X100, 0,5\% NH4OH, Eau dé-ionisée $18 \mathrm{M} \Omega$.

Urine: Les échantillons d'urine sont dilués par un facteur $1: 10$ avec le diluant : $0,1 \%$ Triton $\mathrm{X}-100,0,5 \%$ HNO3 et $0,5 \%$ éthanol. L'ajout d'éthanol permet l'exaltation du signal pour des éléments difficilement ionisables comme As et Se.

\section{Étalons :}

Les échantillons de sang total sont analysés par ajouts dosés sur un « pool» de sang total. Les échantillons 
d'urine sont analysés par étalonnage externe. Les étalons sont préparés avec le même diluant ayant servi pour les échantillons.

\section{Étalons internes :}

Une solution multi-élémentaire contenant $\left({ }^{6} \mathrm{Li},{ }^{45} \mathrm{Sc},{ }^{89} \mathrm{Y}\right.$, ${ }^{115} \mathrm{In},{ }^{103} \mathrm{Rh},{ }^{209} \mathrm{Bi}$ ) est utilisée comme étalon interne.

\section{Résultats et discussions}

\section{Sang Total}

L'étalonnage est effectué par ajouts dosés sur un « pool » de sang total et les échantillons sont analysés à partir de la courbe d'étalonnage ainsi obtenue. Des ajouts de 0,2 , 1,4 et $10 \mu \mathrm{g} / \mathrm{L}$ sont effectués pour les éléments traces.

A l'issu de l'étalonnage, l'échantillon de base de sang total est dopé successivement par des éléments interférents tels que $\mathrm{C}, \mathrm{Ca}$ et $\mathrm{Cl}$ à une concentration de $200 \mathrm{mg} / \mathrm{L}$ afin de valider l'élimination des interférences générées par ces majeurs dans les matrices sang total en mode CRI : Ca (interférence sur ${ }^{78} \mathrm{Se}$ et ${ }^{80} \mathrm{Se}$ ), $\mathrm{Cl}$ (interférences sur ${ }^{51} \mathrm{~V},{ }^{52} \mathrm{Cr},{ }^{77} \mathrm{Se}$ et ${ }^{75} \mathrm{As}$ ) et $\mathrm{C}$ (interférence sur $\left.{ }^{52} \mathrm{Cr}\right) \ldots$

Les concentrations obtenues sont présentées dans le tableau II. Ces résultats ne montrent pas d'augmentation de la concentration mesurée des analytes après ajout d'interférents. Ceci permet de valider l'efficacité de la gestion des interférences spectrales par ajout d'hydrogène au niveau du cône écréteur pour l'ensemble des éléments dosés dans les échantillons.

Un séronorm sang total WB1 est analysé par la suite et les concentrations obtenues sont comparées aux valeurs attendues données par le fournisseur. Les valeurs moyennes obtenues sur 10 analyses de WB1 sont présentées dans le tableau III. Ces résultats montrent une bonne corrélation entre les valeurs mesurées et les valeurs attendues pour l'ensemble des éléments traces et majeurs. Il est à noter également que les valeurs obtenues pour les trois isotopes du Sélénium ${ }^{77} \mathrm{Se},{ }^{78} \mathrm{Se}$ et ${ }^{80} \mathrm{Se}$ subissant des interférences différentes sont cohérentes entre elles, ce qui prouve la gestion simultanée de ces interférences par l'ajout d' $\mathrm{H}_{2}$ au niveau du cône écréteur.

Tableau II : Gestion des interférences en mode CRI, liées à la présence des majeurs $C$, Cl et Ca à une concentration de $200 \mathrm{mg} / \mathrm{L}$.

\begin{tabular}{|l|c|c|c|c|c|}
\hline $\begin{array}{l}\text { Nom } \\
\text { Échantillons }\end{array}$ & $\begin{array}{c}\mathrm{Cr52} \\
\boldsymbol{\mu g} / \mathrm{L}\end{array}$ & $\begin{array}{c}\text { Mn55 } \\
\boldsymbol{\mu g} / \mathrm{L}\end{array}$ & $\begin{array}{c}\mathrm{As75} \\
\boldsymbol{\mu g} / \mathrm{L}\end{array}$ & $\begin{array}{c}\mathbf{Z n 6 6} \\
\boldsymbol{\mu g} / \mathrm{L}\end{array}$ & $\begin{array}{c}\text { Se80 } \\
\boldsymbol{\mu g} / \mathbf{L}\end{array}$ \\
\hline Base & 0,14 & 0,46 & 0,13 & 258,64 & 9,40 \\
\hline Base + Ca & 0,11 & 0,47 & 0,10 & 255,36 & 9,90 \\
\hline Base + Cl & 0,14 & 0,48 & 0,11 & 265,07 & 10,04 \\
\hline Base + C & 0,14 & 0,48 & 0,11 & 262,87 & 10,99 \\
\hline
\end{tabular}

Tableau III : Comparaison valeurs mesurées / valeurs attendues pour l'échantillon de sang total WB1.

\begin{tabular}{|l|c|c|}
\hline Élément & $\begin{array}{c}\text { Valeur cible } \\
\boldsymbol{\mu g} / \mathbf{L}\end{array}$ & $\begin{array}{c}\text { Valeur mesurée } \\
\boldsymbol{\mu g} / \mathbf{L}\end{array}$ \\
\hline $\mathbf{2 7 A l}$ & $13,0-21,2$ & $20,0 \pm 1,7$ \\
\hline $\mathbf{5 1 V}$ & $0,27-0,37$ & $0,29 \pm 0,4$ \\
\hline $\mathbf{5 2 C r}$ & $0,42-0,78$ & $0,42 \pm 0,18$ \\
\hline $\mathbf{5 5 M n}$ & $10,0-11,2$ & $9,8 \pm 0,04$ \\
\hline $\mathbf{5 6 F e} *$ & $404-460$ & $419,9 \pm 0,7$ \\
\hline $\mathbf{6 3 C u}$ & $531-597$ & $574 \pm 6$ \\
\hline $\mathbf{6 6 Z n}$ & $5200-5800$ & $5151 \pm 53$ \\
\hline $\mathbf{7 5 A s}$ & $1,4-2,2$ & $1,8 \pm 0,2$ \\
\hline $\mathbf{8 0 S e}$ & $74,4-85,2$ & $79 \pm 2$ \\
\hline $\mathbf{7 7 S e}$ & $74,4-85,2$ & $79 \pm 3$ \\
\hline $\mathbf{7 8 S e}$ & $74,4-85,2$ & $77,2 \pm 2$ \\
\hline $\mathbf{1 1 1 C d}$ & $0,68-0,80$ & $0,54 \pm 0,04$ \\
\hline $\mathbf{1 3 8 B a}$ & $52,55-52,65$ & $54,0 \pm 0,4$ \\
\hline $\mathbf{P b}$ & $26,2-29,0$ & $27,6 \pm 0,08$ \\
\hline $\mathbf{2 3 8 U}$ & $0,16-0,18$ & $0,17 \pm 0,001$ \\
\hline$*$ concentration en $\mathrm{mg} / \mathrm{L}$ & & \\
\hline & & \\
\hline
\end{tabular}

\section{Urine}

L'analyse de traces et de majeurs est effectuée dans un échantillon de séronorm urine en mode CRI avec $\mathrm{H}_{2}$ à $0,1 \mathrm{~L} / \mathrm{min}$ au cône écréteur. La comparaison des résultats avec et sans CRI (Figure III) permet de mettre en évidence les points suivants :

- possibilité d'analyse simultanée de traces et de majeurs dans une seule séquence analytique

- les résultats obtenus pour les éléments non interférés sont identiques pour les 2 modes avec et sans gaz CRI

- L'ajout de gaz CRI permet la gestion efficace des interférences spectrales observées sur les éléments ${ }^{51} \mathrm{~V} \quad\left({ }^{35} \mathrm{Cl}{ }^{16} \mathrm{O}\right),{ }^{52} \mathrm{Cr} \quad\left({ }^{40} \mathrm{Ar}^{12} \mathrm{C}\right),{ }^{77} \mathrm{Se}\left({ }^{40} \mathrm{Ar}{ }^{37} \mathrm{Cl}\right),{ }^{78} \mathrm{Se}$ $\left({ }^{39} \mathrm{Ar}^{39} \mathrm{Ar}\right)$.

Un test de stabilité effectué sur le NIST 2670a reconstitué et dilué par un facteur 1:10 avant analyse est effectué sur 40 échantillons et sur une durée d'environ 3 heures.

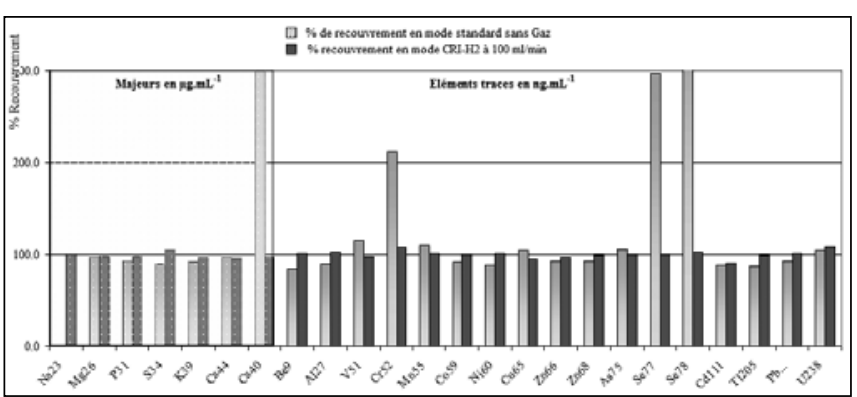

Figure 3 : Pourcentages de recouvrement obtenus avec et sans CRI pour l'échantillon d'urine. 
Les résultats obtenus sur les signaux bruts sans aucune correction par les étalons internes sont présentés dans le tableau IV. Les écart-type relatifs (\% RSD) calculés à partir des 40 valeurs ainsi obtenues sont tous inférieurs à $3 \%$ sur l'ensemble des éléments traces et majeurs et mettent en évidence la stabilité de l'ensemble du système ainsi que l'absence de bouchage des cônes à

Tableau IV : Test de stabilité long terme ( 3 heures) sur un échantillon d'urine NIST 2670a.

\begin{tabular}{|l|c|c|}
\hline \multicolumn{2}{|l|}{$\begin{array}{l}\text { NIST 2670a Niveau haut } \\
\text { dilution 1:10 }\end{array}$} & \% RSD \\
\hline $\mathbf{N a 2 3} \mathbf{~ m g} / \mathbf{L}$ & 80,1 & 1,3 \\
\hline $\mathbf{M g 2 4} \mathbf{~ m g} / \mathbf{L}$ & 2,1 & 1,2 \\
\hline $\mathbf{K 3 9} \mathbf{~ m g} / \mathbf{L}$ & 41,7 & 1,2 \\
\hline $\mathbf{C a} 40 \mathrm{mg} / \mathrm{L}$ & 3,0 & 1,1 \\
\hline $\mathbf{M n 5 5} \mu \mathrm{g} / \mathrm{L}$ & 9,2 & 1,9 \\
\hline $\mathbf{A s 7 5} \mu \mathrm{g} / \mathrm{L}$ & 22,1 & 3,9 \\
\hline $\mathbf{S e 8 0} \mu \mathrm{g} / \mathrm{L}$ & 22,8 & 3,9 \\
\hline $\mathbf{S e 7 7} \mu \mathrm{g} / \mathrm{L}$ & 20,7 & 3,6 \\
\hline $\mathbf{C d 1 1 1} \mu \mathrm{g} / \mathrm{L}$ & 0,43 & 3,6 \\
\hline $\mathbf{T 1 2 0 5} \mu \mathrm{g} / \mathrm{L}$ & 0,5 & 2,7 \\
\hline $\mathbf{P b} . . . \mu \mathrm{g} / \mathrm{L}$ & 24 & 2,8 \\
\hline $\mathbf{U} 238 \mu \mathrm{g} / \mathrm{L}$ & 0,5 & 1,2 \\
\hline
\end{tabular}

l'issue de cette analyse.

\section{Conclusion}

L'interface CRI permet une gestion efficace des interférences polyatomiques observées dans les matrices biologiques pour des éléments tels que ${ }^{52} \mathrm{Cr},{ }^{51} \mathrm{~V},{ }^{75} \mathrm{As}$ et ${ }^{80} \mathrm{Se}$ sans compromettre les performances des éléments non interférés tels que ${ }^{111} \mathrm{Cd},{ }^{205} \mathrm{Tl},{ }^{208} \mathrm{~Pb}$ et ${ }^{238} \mathrm{U}$. L'association du miroir ionique à $90^{\circ}$ et du détecteur offrant une gamme dynamique linéaire de plus de 9 ordres de grandeur permet l'analyse simultanée des éléments traces et majeurs pour une meilleure productivité et une consommation de volume d'échantillon réduite. Ceci présente un avantage majeur pour les applications cliniques où le volume d'échantillon constitue souvent une limitation.

\section{Références}

1. Bonnefoy C., Menudier A., Moesch C., Lachâtre G., Mermet J.M. Validation of the determination of lead in whole blood by ICP-MS. J. Anal. At. Spectrom, 2002 ; $17: 1161-5$.

2. Rodushhkin I., Ödman F., Olofsson A., Axelsson M.D. Determination of 60 elements in whole blood by sector field inductively coupled plasma mass spectrometry. J. Anal. At. Spectrom, 2000 ; 15 : 937-44.

3. Reed N.M., Cairns R.O., Hutton R.C., Takaku Y. Characterization of polyatomic ion interferences in inductively coupled plasma mass spectrometry using a high resolution mass spectrometer. J. Anal. At. Spectrom, 1994 ; 9, 881-96.

4. Krachler M., Alimonti A., Petruccia F., Forastiereban F., Caroli S. Influence of pre-treatment on the determination of trace elements in urine by quadrupole and magnetic sector inductively coupled plasma mass spectrometry. J. Anal. At. Spectrom, $1998 ; 13$ : 701-5.

5. Kalinitchenko I. Ion Optical System for a Mass Spectrometer, US Patent 6614021 B1, 2 December 2003. 